\title{
ON SUPERCOMPLEXES
}

\author{
BY \\ V. K. A. M. GUGENHEIM
}

Introduction. The recent work of Kan (cf. $[1 ; 2]$ ) develops homotopy theory in the context of abstract semi-cubical theory. When one tries to combine this with homology theory an unfortunate fact intervenes: The "models" (defined in analogy to Definition 3 below) turn out not to be acyclic. [To see this, consider the 1-dimensional model, generated by an element $\kappa$, say, in dimension 1. Then, using the notation of Appendix II below, $c=\left(\sigma_{1}+\sigma_{2}\right) \kappa$ is easily seen to be a 2-cycle. All boundaries of 3-dimensional elements are doubly degenerate. Hence $c$ can neither bound nor be homologous to a cycle with a "vertex" as carrier.]

For this and other reasons it seems desirable to use semisimplicial rather than semi-cubical theory. The modifications to be made in Kan's scheme are evident except for one rather awkward theorem which becomes necessary in the treatment of homotopy extension and fibre spaces. The present paper fills this gap (Theorems 3 and $3^{*}$ ).

Kan introduces into abstract semi-cubical theory a condition (the "extension axiom," here referred to as the "Kan condition," see $\$ 2$ below) which expresses within the framework of this theory the geometric fact that the boundary of a cube minus any of its faces is a retract of the cube; and shows that this condition is all that is needed in homotopy theory.

The semi-simplicial analogy is obvious: but is not so immediately applicable to questions of homotopy since one has to begin by translating a condition relating to a "simplex" into one relating to a "prism."

Following a suggestion of S. Eilenberg, however, we deal here with the general case corresponding to the introduction into the theory of arbitrary products of models. This leads to an abstract scheme (supercomplexes) in which homotopy can be dealt with and the theory of fibre spaces and the spectral sequence given in [3] becomes immediately applicable; the idea of defining fibre-spaces in the manner of $\$ 3$ below, an immediate adaptation to the present context of the definition due to J.-P. Serre, was first suggested by D. Kan.

I am indebted to the referee who pointed out that my original version of Definition 8 below was unnecessarily restrictive.

\section{Supercomplexes.}

Definition 1. A semisimplicial complex $A$ is a set graded by non-negative integers (the subset with graduation $n$ being denoted by $A_{n}$ ) together with functions

Received by the editors March 12, 1956 and, in revised form, June 4, 1956. 


$$
\begin{aligned}
\partial_{i}: A_{n} \rightarrow A_{n-1} & (n \geqq 1,0 \leqq i \leqq n), \\
s_{i}: A_{n} \rightarrow A_{n+1} & (n \geqq 0,0 \leqq i \leqq n)
\end{aligned}
$$

which satisfy the following:

$$
\begin{array}{rlr}
\partial_{i} \partial_{j} & =\partial_{j-1} \partial_{i} & (i<j), \\
\partial_{i} s_{j} & =s_{j-1} \partial_{i} & (i<j), \\
& =\text { (identity) } & (i=j \text { or } j+1), \\
& =s_{j} \partial_{i-1} & (i>j+1), \\
s_{i} s_{j} & =s_{j} s_{i-1} & (i>j) .
\end{array}
$$

The graduation is called the dimension.

If $A, B$ are semisimplicial complexes, then a function $f: A \rightarrow B$ such that $f A_{n} \subset B_{n}, f \partial_{i}=\partial_{i} f, f s_{i}=s_{i} f$ is called a semisimplicial map. Semisimplicial complexes and maps form a category denoted by $\delta$.

The notations $\partial_{i}$ are ambiguous; we should write $\partial_{i}^{n}: A_{n} \rightarrow A_{n-1}$, but shall álways dispense with this. A composition of $\partial_{i}$ 's and $s_{j}$ 's is called a semisimplicial operator. A symbol such as

$$
s_{i_{q}} \cdots s_{i_{1}} \partial_{j_{1}} \cdots \partial_{j_{p}}
$$

can thus stand for an infinity of operators according to the dimension of the intended argument: this dimension is referred to as the domain of the operator. We shall also call $q-p$ the height of the above operator.

LEMma 1. Every semisimplicial operator can be written uniquely in the form

$$
s_{i_{q}} \cdots s_{i_{1}} \partial_{j_{1}} \cdots \partial_{j_{p}}
$$

where

$$
i_{1}<\cdots<i_{q}, \quad j_{1}<\cdots<j_{p} .
$$

(The cases $q=0, p=0$ are included and must be interpreted in the obvious way.)

The proof is easy, using relations (1).

Definition 2. Let $A, B$ be semisimplicial complexes. The cartesian product $A \times B$ is a semisimplicial complex defined by $(A \times B)_{n}=A_{n} \times B_{n}$, where the last symbol stands for the set-theoretical product, and

$$
\begin{aligned}
& \partial_{i}(a \times b)=\left(\partial_{i} a \times \partial_{i} b\right) \\
& s_{j}(a \times b)=\left(s_{j} a \times s_{j} b\right) .
\end{aligned} \quad\left(a \in A_{n}, b \in B_{n}\right),
$$

If $f: A \rightarrow A^{1}, g: B \rightarrow B^{1}$ are semisimplicial maps, the semisimplicial map $f \times g: A \times B \rightarrow A^{1} \times B^{1}$ is defined by $(f \times g)(a \times b)=(f a \times g b)$.

Definition 3. For every $n \geqq 0$ we define a semisimplicial complex $\Delta^{n}$ (the " $n$-dimensional model") as follows: Let $\delta^{n}$ be a symbol. Then $\Delta_{k}^{n}$ is to be the set 
consisting of all symbols $\varphi \delta^{n}$ where $\varphi$ is any semisimplicial operator of height $k-n$ and domain $n$; we define

$$
\begin{aligned}
& \partial_{i}\left(\varphi \delta^{n}\right)=\left(\partial_{i} \varphi\right) \delta^{n}, \\
& s_{i}\left(\varphi \delta^{n}\right)=\left(s_{i} \varphi\right) \delta^{n} .
\end{aligned}
$$

Lemma 1 allows us to survey easily the elements of $\Delta^{n}$.

Let $A$ be any semisimplicial complex and $a \in A_{n}$. A unique semisimplicial map

$$
\tilde{a}: \Delta^{n} \rightarrow A
$$

is defined by $a$; namely

$$
\tilde{a}\left(\varphi \delta^{n}\right)=\varphi a .
$$

In particular we define

$$
\begin{aligned}
& \epsilon^{i}: \Delta^{n-1} \rightarrow \Delta^{n} \\
& (0 \leqq i \leqq n), \\
& \eta^{i}: \Delta^{n+1} \rightarrow \Delta^{n} \\
& (0 \leqq i \leqq n)
\end{aligned}
$$

by

$$
\begin{aligned}
\epsilon^{i} \delta^{n-1} & =\partial_{i} \delta^{n}, \\
\eta^{i} \delta^{n+1} & =s_{i} \delta^{n} .
\end{aligned}
$$

The symbols $\partial^{i}, s^{i}, \epsilon^{i}, \eta^{i}$ are now related exactly as in [4], where $F$ is written for our $\partial$, and $D$ for our $s$.

Definition 4. Let $A$ be a semisimplicial complex and $\left(n_{1}, \cdots, n_{k}\right)(k \geqq 1)$ an ordered set of non-negative integers. We define $A\left(n_{1}, \cdots, n_{k}\right)$ to be the set of all semisimplicial maps

$$
u: \Delta^{n_{1}} \times \cdots \times \Delta^{n_{k}} \rightarrow A ;
$$

we shall call $k$ the order and $n_{1}+\cdots+n_{k}$ the dimension of $u$. We define

$$
\begin{aligned}
& \partial_{i}^{t}: A\left(n_{1}, \cdots, n_{t-1}, n_{t}, n_{t+1}, \cdots, n_{k}\right) \\
& \rightarrow A\left(n_{1}, \cdots, n_{t-1}, n_{t}-1, n_{t+1}, \cdots, n_{k}\right)
\end{aligned}
$$

by

$$
\partial_{i}^{t} u=u \circ\left(1 \times \cdots \times 1 \times \epsilon^{i} \times 1 \times \cdots \times 1\right)
$$

where the $\epsilon^{i}$ occurs in the $t$ th place; $1 \leqq t \leqq k ; 0 \leqq i \leqq n_{t}$. A similar definition (using $\eta^{i}$ ) is given for $s_{i}^{t}$.

These operators satisfy the following identities:

$$
\left.\begin{array}{l}
\text { The operators for a given } t \text { satisfy the relations (1). } \\
\text { Operators with differing } t \text { commute. }
\end{array}\right\}
$$


The proof is obvious.

The union of the sets $A\left(n_{1}, \cdots, n_{k}\right)$ together with the operators we have defined we denote by $P A$.

If $f: A \rightarrow B$ is a semisimplicial map, we define

$$
\odot f: P A \rightarrow \odot B
$$

by

$$
(\odot f) u=f u \text {. }
$$

Definition 5. A set graded by ordered sets of non-negative integers and provided with operators satisfying (2) is called a supercomplex; a function $f: A \rightarrow B$, where $A, B$ are supercomplexes, which preserves graduation and commutes with all operators is called a super-map.

The category of supercomplexes and super-maps is denoted by $P S$.

It is easily seen that $P$ can be regarded as a covariant functor

$$
\odot: S \rightarrow \odot S ;
$$

in particular, for any map $f$ of $\delta$, $\odot$ is a super-map.

Let $A$ be a semisimplicial complex. The correspondence $a \rightarrow \tilde{a}$ defined earlier is clearly $1-1$ and onto; also, if $a \in A_{n}$ then $\tilde{a} \in A(n)$ and

$$
\widetilde{\partial_{i} a}=\partial_{i}^{1} \tilde{a}, \quad \widetilde{s_{i} a}=s_{i}^{1} \tilde{a}
$$

as is easily seen. Thus, identifying $a$ with $\tilde{a}, \partial_{i}, s_{i}$ with $\partial_{i}^{1}, s_{i}^{1}$, we can identify $A_{n}$ with $A(n)$ and thus regard $A$ as a subset of $\odot A$; in other words:

Theorem 1. Any semisimplicial complex $A$ can be regarded as the subset of order 1 of the supercomplex $\odot A$.

\section{Kan condition.}

Definition 6. Let $A$ be a supercomplex and $\left(n_{1}, \cdots, n_{k}\right)$ an ordered set of integers. For each integer $x, 1 \leqq x \leqq k$, let $n_{x}>0$ and $e_{x}$ be some subset of $\left[n_{x}\right]\left({ }^{1}\right)$. For each $i \in e_{x}$ let $v_{t}^{x} \in A\left(n_{1}, \cdots, n_{x-1}, n_{x}-1, n_{x+1}, \cdots, n_{k}\right)$ be given. The (indexed) set $S=\left\{v_{t}^{x} \mid 1 \leqq x \leqq k, i \in e_{x}\right\}$ is said to be consistent if

$$
\begin{array}{ll}
\partial_{i}^{x} v_{j}^{x}=\partial_{j-1}^{x} v_{i}^{x} & \left(i<j, v_{i}^{x}, v_{j}^{x} \in S\right), \\
\partial_{i}^{x} v_{j}^{y}=\partial_{j}^{y} v_{i}^{x} & \left(x \neq y, v_{i}^{x}, v_{j}^{y} \in S\right) .
\end{array}
$$

The faces of any $v \in A\left(n_{1}, n_{2}, \cdots, n_{k}\right)$ form a consistent set.

Definition 7. Let the notation be as in Definition 6 and let $a_{x}=\left[n_{x}\right]-e_{x}$. If, given the set $S$, there is $v \in A\left(n_{1}, \cdots, n_{k}\right)$ such that

$$
\partial_{i}^{x} v=v_{i}^{x}
$$

whenever ${ }_{i}^{x} \in S$

(1) $[n]$ denotes the set $\{0,1,2, \cdots, n\}$. 
then we say that $A$ satisfies the condition $K\left(n_{1}, a_{1} ; \cdots ; n_{k}, a_{k}\right)$.

We shall sometimes call $v_{i}^{x}$ the "given faces" and $\partial_{j}^{y} v, i \leqq y \leqq k, j \in a_{y}$, the "missing faces."

Definition 8. The supercomplex $A$ will be called a Kan-complex (or said to satisfy the Kan condition) if it satisfies $K\left(n_{1}, a_{1} ; \cdots ; n_{k}, a_{k}\right)$ whenever $k \geqq 1$ and

(i) $n_{x}>0,1 \leqq x \leqq k$;

(ii) There is at least one $x, 1 \leqq x \leqq k$, such that $a_{x}$ is a nonempty proper subset of $\left[n_{x}\right]$.

Putting $k=1, \partial_{i}=\partial_{i}^{1}$ (as in Theorem 1) these definitions apply to semisimplicial complexes.

The rest of this paper is largely devoted to the proof of the following two theorems:

TheOREM 2. Let $A$ be a semisimplicial complex satisfying $K(n ;\{0\})$ and $K(n ;\{n\})$ for all $n>0$. Then $A$ satisfies Kan's condition.

An analogous theorem in the case of the semi-cubical theory is due to Kan, cf. $[1 ; 2]$.

TheOREM 3. If the semisimplicial complex A satisfies Kan's condition, so does the supercomplex $\odot A$.

The proof of Theorem 3 will be given in Chapter 5 ; that of Theorem 2 follows now. It begins with two lemmas:

Lemma 2. Let $n>0$ and $a \subset[n]$ be a nonempty subset containing at most $n-1$ integers, let $i \in[n]-a$ and let $a^{\prime} \subset[n-1]$ be obtained from a by decreasing all integers $>i$ by 1 . If the semisimplicial complex $A$ satisfies $K(n ; a)$ and $K\left(n-1 ; a^{\prime}\right)$, then it also satisfies $K(n ; a \cup\{i\})$. fine

Proof. Let the set $S=\left\{v_{j} \mid v_{j} \in A_{n-1}, j \in[n]-a-\{i\}\right\}$ be consistent. De-

$$
\begin{aligned}
w_{j} & =\partial_{i-1} v_{j} \text { when } j<i, j \in[n]-a \\
& =\partial_{i} v_{j+1} \text { when } j \geqq i, j+1 \in[n]-a .
\end{aligned}
$$

It is easily verified that $\left\{w_{j} \mid j \in[n-1]-a^{\prime}\right\}$ is consistent. Hence, by $K\left(n-1 ; a^{\prime}\right)$, there is $v_{\imath} \in A_{n}$ such that $w_{j}=\partial_{j} v_{i}, j \in[n-1]-a^{\prime}$. Then it is easily verified that $S \cup_{v_{i}}$ is consistent. Hence, by $K(n ; a)$ there is $v \in A_{n+1}$ such that $v_{j}=\partial_{j} v, j \in[n]-a$.

Lemma 3. Let $n>0$ and $z \in[n]$. Let $A$ be a semisimplicial complex which satisfies

(i) $K(m ; c)$ for every $m<n$ and every nonempty proper subset $c$ of $[m]$.

(ii) $K(n ;\{z\})$.

Then it also satisfies $K(n ; b)$ where $b$ is any nonempty proper subset of $[n]$ such that $z \in b$. 
Proof. Let $p$ be the number of elements in $b$. If $p=1, b=\{z\}$, and $K(n ; b)$ is given. Now we use an induction on $p$. Thus, let $p>1, i \in b, i \neq z$. Let $a=b-\{i\}$. Then, by the inductive hypothesis and (i), $K(n ; a)$ and $K(n-1$; $\left.a^{\prime}\right)$ are true, where $a^{\prime}$ means the same as in Lemma 2. Hence $K(n ; a \cup\{i\})$ $=K(n ; b)$ is true.

In view of Lemma 3, Theorem 2 will be proved, by an induction on $n$, if, supposing that $A$ satisfies $K(n ;\{0\})$ and $K(n ;\{n\})$ for all $n>0$ as well as (i) of Lemma 3 , then it also satisfies $K(n ;\{z\})$ for $z \in[n]$.

For $z=0, z=n$, this is clear. For $0<z<n$, we use an induction on $z$. Thus, let $v_{0}, \cdots, v_{z-1}, v_{z+1}, \cdots, v_{n} \in A_{n-1}$ be given, and consistent.

We begin by finding

$$
w_{0}, w_{1}, \cdots, w_{n} \in A_{n}
$$

which are to satisfy the consistency condition as well as

$$
\partial_{n} w_{i}=v_{i} \text { if } i \neq z .
$$

We define $w_{n}=s_{n-1} v_{n}$. Suppose now that $w_{i}$ has been found for $k<i \leqq n$ where $k>z$. Then $w_{k}$ has to satisfy

$$
\partial_{j} w_{k}=\partial_{k} w_{j+1}=u_{j} \text { say }
$$

for $k \leqq j<n$; as well as

$$
\partial_{n} w_{k}=v_{k}=u_{n} \text { say. }
$$

It is easily verified that $u_{k+1}, \cdots, u_{n} \in A_{n-1}$ satisfy the consistency condition; hence (due to Lemma 3 in the case $z=0$ ) the required $w_{k}$ exists.

Thus, we find $w_{z+1}, \cdots, w_{n}$.

Next, suppose that $w_{i}$ has also been found for $0 \leqq i<l<z$. Then $w_{l}$ must satisfy

$$
\begin{aligned}
& \partial_{i} w_{l}=\partial_{l-1} w_{i}=u_{i} \text { say, for } 0 \leqq i<l<z, \\
& \partial_{j} w_{l}=\partial_{l} w_{j+1}=u_{j} \text { say, for } z \leqq j<n, \\
& \partial_{n} w_{l}=v_{l}=u_{n} \text { say. }
\end{aligned}
$$

It is easily seen that $u_{0}, \cdots, u_{l-1}, u_{z}, \cdots, u_{n} \in A_{n-1}$ satisfy the consistency condition. Hence (due to Lemma 3 with $z-1$ in place of $z$ and the inductive hypothesis) the required $w_{l}$ exists.

Finally, $w_{z}$ must satisfy

$$
\begin{aligned}
& \partial_{i} w_{z}=\partial_{z-1} w_{i}=u_{i} \text { say for } 0 \leqq i<z, \\
& \partial_{j} w_{z}=\partial_{z} w_{j+1}=u_{j} \text { say for } z \leqq j<n,
\end{aligned}
$$

where, again, $u_{0}, \cdots, u_{n-1} \in A_{n-1}$ satisfy the consistency conditions. Hence, by $K(n ;\{n\})$, the required $w_{z}$ exists.

Hence, by $K(n+1,\{n+1\})$, there is $u \in A_{n+1}$ such that 


$$
\partial_{i} u=w_{i} \text { where } 0 \leqq i \leqq n .
$$

Let $v=\partial_{n+1} u$. Then, for $i \in[n]-\{z\}$

$$
\partial_{i} v=\partial_{i} \partial_{n+1} u=\partial_{n} \partial_{i} u=\partial_{n} w_{i}=v_{i},
$$

and the proof of Theorem 2 is complete.

\section{Fibre maps.}

Definition 9. Let $A, B$ be supercomplexes and $f: A \rightarrow B$ a super-map. We say that $f$ satisfies the condition $K\left(n_{1}, a_{1} ; \cdots ; n_{k}, a_{k}\right)$ (cf. Definitions 6 and 7 for the notation) if the following is true: Given the set $S$ satisfying the same conditions as in Definitions 6 and 7 and given also $u \in B\left(n_{1}, \cdots, n_{k}\right)$ such that

$$
\partial_{i}^{x} u=f v_{i}^{x}
$$

whenever $v_{i}^{x} \in S$

then there is $v \in A\left(n_{1}, \cdots, n_{k}\right)$ such that

$$
\partial_{i}^{x} v=v_{i}^{x}
$$

whenever $\boldsymbol{v}_{i}^{x} \in S$

and $f v=u$.

Definition 10. We say that the super-map $f: A \rightarrow B$ is a fibre map if it satisfies the condition $K\left(n_{1}, a_{1} ; \cdots ; n_{k}, a_{k}\right)$ whenever conditions (i) and (ii) of Definition 8 are true.

With the identification of Theorem 1, these definitions apply in an obvious way to the case of semisimplicial maps.

TheOREm $2 *$. Let $f$ be a semisimplicial map satisfying $K(n ;\{0\})$ and $K(n ;\{n\})$ for all $n>0$. Then $f$ is a fibre map. Pf.

THEOREM 3*. If the semisimplicial map $f$ is a fibre map, so is the super-map

Only a few words need be added to the proof of Theorem 3 below in order to get Theorem $3 *$.

The proof of $2^{*}$, similarly, is essentially the same as that of Theorem 2 above: In the proof of Lemma 2, note that we are also given $u$ such that $f v_{j}=\partial_{j} u$. Hence $f w_{j}=\partial_{i-1} f v_{j}=\partial_{i-1} \partial_{j} u=\partial_{j}\left(\partial_{i} u\right)$ when $j<i$ and $f w_{j}=\partial_{i} f v_{j+1}$ $=\partial_{i} \partial_{j+1} u=\partial_{j}\left(\partial_{i} u\right)$ when $j \geqq i$. Hence, again, $v_{i}$ exists, satisfying $f v_{i}=\partial_{i} u$.

Nothing is changed in the proof of Lemma 3 , but similar additions must be made in the main proof. We have given a $U$ such that $f v_{i}=\partial_{i} U$; and we require of the $w_{i}$ the additional condition $f w_{i}=\partial_{i} s_{n} U$. Thus $f w_{n}=U$, and the required $w_{n}$ exists by an application of Lemma 3 with $z=0$; (not even $w_{n}$ can be "written down" in this case). Similar "additional requirements" are easily written down in the rest of the proof, and the $u$ we finally obtain satisfies $f u=s_{n} U$. Hence $f v=f \partial_{n+1} u=\partial_{n+1} s_{n} U=U$, as required.

Theorem $3 *$ applies, in particular, to the "prisms" of $A(1, n)$ and $A(1,1, n)$. 
This makes it possible to apply the entire theory of the spectral sequence of [3] to the case of semisimplicial fibre-spaces. The use of the "cubical" terminology will be explained in Appendix II below.

4. Partitioning.

Definition 11 (cf. [4]). Let

$$
\tau=\left(\nu_{1}, \cdots, \nu_{q} ; \mu_{1}, \cdots, \mu_{p}\right)
$$

be a permutation of $[p+q-1]$ such that $\nu_{1}<\cdots<\nu_{q}, \mu_{1}<\cdots<\mu_{p}$. Then we call $\tau$ a $(p, q)$ shuffle. With $\tau$ we associate the semisimplicial operators

$$
\begin{aligned}
& s_{\nu}=s_{\nu q} s_{\nu q-1} \cdots s_{\nu_{1}}, \\
& s_{\mu}=s_{\mu_{p}} s_{\mu \cdot-1} \cdots s_{\mu_{1}} .
\end{aligned}
$$

With the $(p, q)$ shuffle $\tau$ we can also associate the element

$$
\delta_{\tau}^{p+q}=\left(s_{\nu} \delta^{p}, s_{\mu} \delta^{q}\right) \in \Delta^{p} \times \Delta^{q}(\text { cf. Chapter } 1) .
$$

Lemma 4. Every element of $\Delta^{p} \times \Delta^{q}$ has the form $\varphi \delta_{\tau}^{p_{\tau}^{+q}}$, where $\varphi$ is a suitable semisimplicial operator, and $\tau$ is some $(p, q)$ shuffle.

Proof. Using relations (1), any semisimplicial operator can be written (in several ways!) in the form

$$
\partial_{j_{1}} \cdots \partial_{j a} s_{i b} \cdots s_{i_{1}}
$$

where

$$
j_{1}<\cdots<j_{a}, \quad i_{1}<\cdots<i_{b} .
$$

Hence, any element of $\Delta^{p} \times \Delta^{q}$ has the form

$$
x=\left(\partial_{j_{1}} \cdots \partial_{j_{a} s_{i b}} \cdots s_{i_{1}} \delta^{p}, \partial_{l_{1}} \cdots \partial_{l_{c}} s_{k_{d}} \cdots s_{k_{1}} \delta^{q}\right)
$$

where $j_{1}<\cdots<j_{a}, i_{1}<\cdots<i_{b}, l_{1}<\cdots<l_{c}, k_{1}<\cdots<k_{d}$. If $j_{1}=l_{1}$, $x=\partial_{j_{1}} x^{\prime}$, where the number of $\partial$ 's occurring in the expression of $x^{\prime}$ is less (by 2) than that in $x$. If $j_{1}<l_{1}$, say, notice that

$$
\partial_{l_{1}}=\partial_{l_{1}} \partial_{j_{1}} s_{j_{1}}=\partial_{j_{1}} \partial_{l_{1}+1} s_{j_{1}}
$$

and $x=\partial_{j_{1}} x^{\prime}$ where the expression for $x^{\prime}$ contains one less $\partial$ than that for $x$. Continuing this way, any element of $\Delta^{p} \times \Delta^{q}$ is obtained by successive boundary operations from an element of the form

$$
x=\left(s_{i b} \cdots s_{i_{1}} \delta^{p}, s_{k_{d}} \cdots s_{k_{1}} \delta^{q}\right)\left\{\begin{array}{l}
i_{1}<\cdots<i_{b}, \\
k_{1}<\cdots<k_{d} .
\end{array}\right.
$$

Again, if any $i$ equals any $k$, the corresponding $s$ can be "brought in front" and then be "taken outside."

Hence any element can be obtained by applying simplicial operations to an element of the above form, where the $i$ 's and $k$ 's are all distinct. Now, we must have 


$$
\begin{gathered}
p+b=q+d=N, \text { say; } \\
i_{1}<\cdots<i_{b}<N, \quad k_{1}<\cdots<k_{d}<N .
\end{gathered}
$$

Now, suppose in the interval $0,1, \cdots, N-1$ there is any number, $n$ say, which is neither an $i$ nor a $k$. Then $x=\partial_{n+1} x^{\prime}$, where $x^{\prime}$ has the same form as $x$, all the $i$ 's and $k$ 's above $n$ having been increased by 1 , and then $s_{n}, s_{n+1}$ respectively having been inserted.

In this way, finally we can restrict ourselves to elements $x$ of the above form for which $i_{1}, \cdots, i_{b}, k_{1}, \cdots, k_{d}$ is a permutation of the integers from 0 to $N-1$. But then

$$
b+d=N=p+b=q+d .
$$

Hence $b=q, d=p$; and the lemma is proved.

It follows from Lemma 2 that, given a semisimplicial complex $A$, a map $f: \Delta^{p} \times \Delta^{q} \rightarrow A$ is uniquely defined by the elements $f \delta_{\tau}^{p+q}=a_{r}$, say, for every $(p, q)$-shuffle $\tau$. If the elements $a_{\tau} \in A$ are to define such a map it is necessary and sufficient that

$$
\partial_{i} a_{\sigma}=\partial_{j} a_{\tau}
$$

whenever

$$
\partial_{i} \delta_{\sigma}^{p+q}=\partial_{j} \delta_{\tau}^{p+q} .
$$

The required information is contained in Lemma 5.

Let $\tau=\left(\nu_{1}, \cdots, \nu_{q}, \mu_{1}, \cdots, \mu_{p}\right)$ be a given $(p, q)$-shuffle, let $0 \leqq i \leqq p+q$, $0 \leqq r \leqq q, 0 \leqq s \leqq p$. We say that the ordered set $(i, r, s)$ is of $\tau$-type $1,2,3$ or 4 according as

1.

$$
\nu_{r}+1<i<\nu_{r+1}\left(\text { or } i<\nu_{1} \text {, if } r=0, i>\nu_{q}+1 \text { if } r=q\right. \text { ) }
$$

2.

$$
\begin{array}{r}
i=\mu_{s} \quad\left(\text { or } i=\mu_{p}+1=p+q, r=q, s=p\right), \\
\mu_{s}+1<i<\mu_{s+1}\left(\text { or } i<\mu_{1} \text {, if } s=0, i>p+1 \text { if } s=p\right)
\end{array}
$$$$
i=\nu_{r} \quad\left(\text { or } i=\nu_{q}+1=p+q, s=p, r=q\right) \text {, }
$$

3.

$$
i=\nu_{r}=\mu_{\mathrm{s}}+1 \text {, }
$$

4.

$$
i=\mu_{\varepsilon}=\nu_{r}+1 \text {. }
$$

It is clear that, given $\tau$ and $i, r$ and $s$ exist so as to fulfill the conditions of exactly one of these 4 cases; thus we can say that $i$ is of exactly one of the $4 \tau$-types.

If $i$ is of $\tau$-type 1 , we define the $(p-1, q)$-shuffle $\tau / i$ by

$$
\tau / i=\left(\nu_{1}, \cdots, \nu_{r}, \nu_{r+1}-1, \cdots, \nu_{q}-1 ; \mu_{1}, \cdots, \mu_{s-1}, \mu_{s+1}-1, \cdots, \mu_{p}-1\right)
$$

and if $i$ is of $\tau$-type 2, we define the (p, $q-1)$-shuffle $\tau / i$ by $\tau / i=\left(\nu_{1}, \cdots, \nu_{r-1}, \nu_{r+1}-1, \cdots, \nu_{q}-1, \mu_{1}, \cdots, \mu_{s}, \mu_{s+1}-1, \cdots, \mu_{p}-1\right)$. 
With these notations the following are easily verified:

$$
\begin{aligned}
\partial_{i} \delta_{\tau}^{p+q}= & \left(\epsilon^{i-r} \times 1\right) \delta_{\tau / i}^{p+q-1}(\text { Case } 1), \\
\partial_{i} \delta_{\tau}^{p+q}= & \left(1 \times \epsilon^{1-s}\right) \delta_{\tau / i}^{p+q-1}(\text { Case } 2), \\
\partial_{i} \delta_{\tau}^{p+q}= & \left(s_{\nu_{q}-1} \cdots s_{\nu_{r+1}-1} s_{\nu_{r-1}} \cdots s_{\nu_{1}} \delta^{p}, s_{\mu_{p}-1}\right. \\
& \left.\cdots s_{\mu_{s+1}-1} s_{\mu_{s-1}} \cdots s_{\mu_{1}} \delta^{q}\right) \text { (Cases 3 and 4). }
\end{aligned}
$$

If $(i, r, s)$ is of $\tau$-type 3 or $4, \mu_{s}$ and $\nu_{r}$ are consecutive integers; we define the $(p, q)$-shuffle $\tau(i)$ by exchanging the values of $\mu_{s}$ and $\nu_{r}$. Thus, if $(i, r, s)$ is of $\tau$-type 3 (4), then it is of $\tau(i)$ type $4(3)$; and $\tau(i)(i)=\tau$; and from (4) it is clear that

$$
\partial_{i} \delta_{\tau}^{p+q}=\partial_{i} \delta_{\tau(i)}^{p+q} .
$$

Lemma 5. Let $\sigma, \tau$ be $(p, q)$-shufles and let

$$
\partial_{i} \delta_{\sigma}^{p+q}=\partial_{j} \delta_{\tau}^{p+q} .
$$

Then $i=j$ and

(i) if $i$ is of $\sigma$-type 1 or $2, \tau=\sigma$,

(ii) if $i$ is of $\sigma$-type 3 or $4, \tau=\sigma$ or $\sigma(i)$.

The proof of this lemma is tedious, and given in Appendix I. Notice that it expresses (essentially) the fact that the partition of $\Delta^{p} \times \Delta^{q}$ by the $\delta_{\tau}^{p+q}$ is a pseudomanifold.

With every $(p, q)$-shuffle $\tau$ we associate the semisimplicial map $\tau_{*}: \Delta^{p+q}$ $\rightarrow \Delta^{p} \times \Delta^{q}$ given by $\tau_{*} \delta^{p+q}=\delta_{\tau}^{p+q}$.

Let $A$ be a semi-simplicial complex and $u \in A\left(n_{1}, \cdots, n_{k}\right)$, where $k \geqq 2$. For every $\left(n_{1}, n_{2}\right)$-shuffle $\tau$ we define $\tau^{*} u \in A\left(n_{1}+n_{2}, n_{3}, \cdots, n_{k}\right)$ by

$$
\tau^{*} u=u \circ\left(\tau_{*} \times 1 \cdots \times 1\right): \Delta^{n^{\prime}+} \Delta^{n_{1} n_{2}} \times \cdots \times \Delta^{n_{k}} \rightarrow A .
$$

A short calculation transforms (3) and (5) into:

$$
\begin{aligned}
\partial_{i}^{1} \tau^{*} & =(\tau / i)^{*} \partial_{i-r}^{1} \text { if }(i, r, s) \text { is of } \tau \text {-type } 1 \\
& =(\tau / i)^{*} \partial_{i-s}^{2} \text { if }(i, r, s) \text { is of } \tau \text {-typé } 2, \\
\partial_{i} \tau^{*} & =\partial_{i}^{1}(\tau(i))^{*} \text { if } i \text { is of } \tau \text {-type } 3 \text { or } 4 .
\end{aligned}
$$

The following is almost immediate:

$$
\partial_{i}^{t} \tau^{*}=\tau^{*} \partial_{i}^{t+1} \text { if } t>1 .
$$

Lemma 6. (i) Given $j, 0 \leqq j \leqq n_{1}$ and an $\left(n_{1}-1, n_{2}\right)$ shuffle $\sigma$, there is a unique $\left(n_{1}, n_{2}\right)$-shuffle $\tau$ and a unique $i$ of $\tau$-type 1 , such that $\sigma=\tau / i$ and $\partial_{i}^{1} \tau^{*}=\sigma^{*} \partial_{j}^{1}$.

(ii) Given $j, 0 \leqq j \leqq n_{2}$ and an $\left(n_{1}, n_{2}-1\right)$ shuffe $\sigma$, there is a unique $\left(n_{1}, n_{2}\right)$ shuffle $\tau$ and $a$ unique $i$ of $\tau$-type 2 , such that $\sigma=\tau / i, \partial_{i}^{1} \tau^{*}=\sigma^{*} \partial_{j}^{2}$.

(iii) If $i$ is of $\tau$-type 3 or 4 and $\partial_{i}^{1} \tau^{*}=\partial_{j}^{1} \sigma^{*}$ then $i=j$ and $\sigma=\tau$ or $\sigma=\tau(i)$. 
Proof. (i) The existence of $\tau, i$ such that $\partial_{i}^{1} \tau^{*}=\sigma^{*} \partial_{j}^{1}$ is immediate from relations (1); the uniqueness assertions follow from Lemma 5 and Equation $3 *$.

(ii) Similarly.

(iii) From Lemma 5 and equation $5^{*}$.

5. Proof of Theorem 3. Let the set of integers $n_{x}, 1 \leqq x \leqq k, n_{x}>0$ and the subsets $a_{x} \subset\left[n_{x}\right]$ be given so as to satisfy the conditions of Definition 8 . The theorem will be proved by an induction on $k$ : For $k=1$, there is nothing to be proved, cf. Theorem 1. Now, we use the partition-operators $\tau^{*}$ of $\$ 4$.

Our process is as follows: For each $\left(n_{1}, n_{2}\right)$-shuffle $\tau$ we find $\tau^{*} v$ so as to be compatible with all the given faces as well as those of the $\sigma^{*} v$ which have been found previously, $\sigma$ being some other $\left(n_{1}, n_{2}\right)$-shuffle; to prove the existence of $\tau^{*} v$ we apply the inductive hypothesis to $A\left(n_{1}+n_{2}, n_{3}, \cdots, n_{k}\right)$.

In order to carry out this programme, we order the $\left(n_{1}, n_{2}\right)$-shuffles lexicographically; thus (we shall write $n_{1}=p, n_{2}=q$ ), if

$$
\begin{aligned}
\tau & =\left(\nu_{1}, \cdots, \nu_{q} ; \mu_{1}, \cdots, \mu_{p}\right), \\
\tau^{\prime} & =\left(\nu_{1}^{\prime}, \cdots, \nu_{q}^{\prime} ; \mu_{1}^{\prime}, \cdots, \mu_{p}^{\prime}\right)
\end{aligned}
$$

then we write $\tau<\tau^{\prime}$ if $\nu_{i}=\nu_{i}^{\prime}$ for $i<j$ and $\nu_{j}<\nu_{j}^{\prime}$.

According to circumstances we shall take the various shuffles either in lexicographical or in inverse lexicographical order. We must arrange matters such that for each successive $\tau^{*} v$ to be found the "given" faces of at least one type (i.e., belonging to one $x$ ) are a nonempty proper subset of all such faces. If the $x$ satisfying condition (ii) of Definition 8 also satisfies $x>2$, then by Equation 6, this condition is satisfied for each $\tau^{*} v$ also, the integer in question being $x-1$.

There remain the cases $x=1,2$.

The. first and last shuffles in the lexicographical order are

$$
\begin{aligned}
\tau_{f} & =(0,1, \cdots, q-1 ; q, q+1, \cdots, p+q-1), \\
\tau_{l} & =(p, p+1, \cdots, p+q-1 ; 0,1, \cdots, p-1)
\end{aligned}
$$

respectively. The successor of each $(p, q)$-shuffle (except $\left.\tau_{l}\right)$ is of the form $\tau(i)$ for some $i$; and so is the predecessor of each face except $\tau_{f}$. Hence, by Lemma 6 , (iii), the required condition is satisfied, with $x=1$, by each successive $\tau^{*} v$ that is determined, except possibly $\tau_{f}^{*} v$ and $\tau_{l}^{*} v$.

We have, by equation $3^{*}$,

$$
\begin{aligned}
\partial_{i}^{1} \tau_{f}^{*} & =\left(\tau_{f} / i\right)^{*} \partial_{i}^{2} \\
& =\left(\tau_{f} / i\right)^{*} \partial_{i-q}^{1} \\
\partial_{i}^{1} \tau_{l} * & =\left(\tau_{l} / i\right)^{*} \partial_{i}^{1} \\
& =\left(\tau_{l} / i\right)^{*} \partial_{i-p}^{2}
\end{aligned}
$$

$$
\begin{aligned}
0 & \leqq i \leqq q-1, \\
q+1 & \leqq i \leqq p+q, \\
0 & \leqq i \leqq p-1, \\
p+1 & \leqq i \leqq p+q .
\end{aligned}
$$

Let $x=1$ (in condition (ii) of Definition 8). Unless $[p]-a_{1}=\{0\}, \tau_{f}^{*} v$ has 
got a given face $\partial_{i}^{1}$; and unless $a_{1}=\{p\}, \tau_{l}{ }^{*} v$ has a missing face of type $\partial_{i}^{1}$. And we can proceed in lexicographical order. In the two excepted cases, $\tau_{l}{ }^{*} v$ has a given face, and $\tau_{f}^{*} v$ a missing one: And we proceed in antilexicographical order. The case $x=2$ is similar.

Finally, in order to complete the inductive proof, we must show that at each stage of the process all the "given faces" form a consistent set. This involves us in some rather cumbersome computations. We deal with the lexicographical case, the antilexicographical one being strictly analogous.

Thus, suppose we have the data of Definition 6, and that $\sigma^{*} v$ has been determined when $\sigma<\tau$, in such a way that all consistency relations are satisfied.

We now make a list of the given faces of $\tau^{*} v$, writing $w_{i}^{x}=\partial_{i}^{x} \tau^{*} v$. If we write such a symbol as $v_{j}^{y}$ we imply that it is one of the given faces.

Let $(i, r, s)$ be of $\tau$-type 1 ; then $w_{i}^{1}=\partial_{i}^{1} \tau^{*} v=(\tau / i)^{*} \partial_{i-r}^{1} v=(\tau / i)^{*} v_{i-r}^{1}$ if $v_{t-1}^{1}$ is given. In this way we find 5 types of given faces:

$$
\begin{aligned}
& \text { I } w_{i}^{1}=(\tau / i) v^{1} v_{i-r} \text { if }(i, r, s) \text { is of } \tau \text {-type } 1 \text {, } \\
& \text { II } w_{i}^{1}=(\tau / i) *_{v_{-s}}^{2} \text { if }(i, r, s) \text { is of } \tau \text {-type 2, } \\
& \text { III } w_{i}^{1}=\partial_{i}^{1}(\tau(i)) *_{v} \text { if } \tau(i)<\tau \text { and } i \text { is of } \tau \text {-type } 3 \text {, } \\
& \text { IV } w_{i}^{1}=\partial_{i}^{1}\left(\tau(i) *_{v} \text { if } \tau(i)<\tau \text { and } i \text { is of } \tau \text {-type } 4\right. \text {, } \\
& \mathrm{V} w_{i}^{x}=\tau^{*} v_{i}^{x+1} \quad \text { if } x>1 .
\end{aligned}
$$

Lemma 6 ensures that this list is complete.

Now, if $i$ is of $\tau$-type $4, \tau(i)>\tau$; and hence case IV is impossible. Let $w_{i}^{1}, w_{j}^{x}$ belong to cases I and V, say. Then, associated with this pair is a consistency relation of "type (I, V)." In this way we obtain 10 types of consistency relation to verify:

$(\mathrm{V}, \mathrm{V})$ : Let $x>1, y>1, i<j$.

$$
\begin{aligned}
& \partial_{i}^{x} w_{j}^{x}=\partial_{i}^{x} \tau^{*} v_{j}^{x+1}=\tau^{*} \partial_{i}^{x+1} v_{j}^{x+1}=\tau^{*} \partial_{j-1}^{x+1} v_{i}^{x+1}=\partial_{j-1}^{x} \tau^{*} v_{i}^{x+1}=\partial_{j-1}^{x} w_{i}^{x} . \\
& \partial_{i}^{x} w_{j}^{y}=\partial_{i}^{x} \tau^{*} v_{j}^{y+1}=\tau^{*} \partial_{i}^{x+1} v_{j}^{y+1}=\tau^{*} \partial_{j}^{y+1} v_{i}^{x+1}=\partial_{j}^{y}{ }^{*} \tau v_{i}^{x+1}=\partial_{j}^{y} w_{i}^{x} .
\end{aligned}
$$

$(\mathrm{I}, \mathrm{V})$ : Let $(i, r, s)$ be of $\tau$-type $1, x>1$.

$$
\partial_{i}^{1} w_{j}^{x}=\partial_{i}^{1} \tau^{*} v_{j}^{x+1}=(\tau / i)^{*} \partial_{i-r}^{1} v_{j}^{x+1}=(\tau / i)^{*} \partial_{j}^{x+1} v_{i-r}^{1}=\partial_{j}^{x}(\tau / i)^{*} v_{i-r}^{1}=\partial_{j}^{x} w_{i}^{1}
$$

$(\mathrm{II}, \mathrm{V})$ is similar.

(III, V): Let $i$ be of $\tau$-type $3, \tau(i)<\tau$ and $x>1$.

$$
\partial_{i}^{1} w_{j}^{x}=\partial_{i}^{1} \tau^{*} v_{j}^{x+1}=\partial_{i}^{1} \tau(i)^{*} v_{j}^{x+1}=\partial_{i}^{1} \partial_{j}^{x} \tau(i)^{*} v=\partial_{j}^{x} \partial_{i}^{1} \tau(i)^{*} v \partial_{j}^{x} w_{i}^{1}
$$

$(\mathrm{I}, \mathrm{I}):$ Let $(i, r, s),(j, t, u)$ both be of $\tau$-type 1 , and $i<j$. Then $i-r<j-t$, $(i, r, s)$ is of $\tau / j$-type $1,(j-1, t, u-1)$ is of $\tau / i$-type 1 and $\tau / j / i=\tau / i / j-1$; all these statements are easily verified. Hence: 


$$
\begin{aligned}
\partial_{i}^{1} w_{j}^{1} & =\partial_{i}^{1}(\tau / j) *^{*} v_{j-t}^{1}=(\tau / j / i)^{*} \partial_{i-r}^{1} v_{j-t}^{1} \\
& =(\tau / i / j-1)^{*} \partial_{j-1-t}^{1} v_{i-r}^{1} \\
& =\partial_{j-1}^{1}(\tau / i) v_{i-r}^{1}=\partial_{j-1}^{1} w_{i}^{1} .
\end{aligned}
$$

The case (II, II) is similar and the case (I, II) only very slightly different.

(I, III): Let $i<j$, let $(i, u, t)$ be of $\tau$-type 1 and $(j, r, s)$ of $\tau$-type 3. We must distinguish 2 cases. First, let $i<j-1$. Then the calculation runs as follows:

$$
\begin{aligned}
\partial_{i}^{1} w_{j}^{1} & =\partial_{i}^{1} \partial_{j}^{1}(\tau(j))^{*} v=\partial_{j-1}^{1} \partial_{i}^{1}(\tau(j))^{*} v \\
& =\partial_{j-1}^{1}(\tau(j) / i)^{*} v_{i-u}^{1}=\partial_{j-1}^{1}((\tau / i)(j-1))^{*} v_{i-u}^{1} \\
& =\partial_{j-1}^{1}(\tau / i) v_{i-u}^{1}=\partial_{j-1}^{1} w_{i}^{1} .
\end{aligned}
$$

The various verifications of "type" required in this computation are easily made. Now, let $i=j-1$. Then the computation runs like this:

$$
\begin{aligned}
\partial_{i}^{1} w_{j}^{1} & =\partial_{i}^{1} \partial_{j}^{1}(\tau(j))^{*}=\partial_{j-1}^{1} \partial_{i}^{1}(\tau(j)) *_{v} \\
& =\partial_{j-1}^{1} \partial_{i}^{1}(\tau(j)(i))^{*} v_{v}^{1}=\partial_{i}^{1} \partial_{j}^{1}(\tau(j)(i))^{*} v_{v} \\
& =\partial_{i}^{1}(\tau(j)(i) / j)^{*} v_{j-r}^{1}=\partial_{i}^{1}(\tau / i)(i)^{*} v_{j-r}^{1} \\
& =\partial_{i}^{1}(\tau / i)^{*} v_{j-r}^{1}=\partial_{j-1}^{1}(\tau / i)^{*} v_{i-r+1}^{1} \\
& =\partial_{j-1}^{1} w_{i}^{1} .
\end{aligned}
$$

Again, several verifications concerning "type" are left to the reader.

Under the heading (I, III) the case $i>j$ is similar; and so are the calculations under (II, III).

(III, III): Let $i<j$ and let $i, j$ each be of $\tau$-type 3 . Then it is easily seen that, in fact, $i<j-1$; and we compute:

$$
\begin{aligned}
\partial_{i}^{1} w_{j}^{1} & =\partial_{i}^{1} \partial_{j}^{1}(\tau(j)) *_{v}=\partial_{j-1}^{1} \partial_{i}^{1}(\tau(j)) *_{v} \\
& =\partial_{j-1} \partial_{i}^{1}(\tau(j)(i))_{v}=\partial_{j-1} \partial_{i}^{1}(\tau(i)(j))^{*} v \\
& =\partial_{i}^{1} \partial_{j}^{1}(\tau(i)(j))^{*} v=\partial_{i}^{1} \partial_{j}^{1}(\tau(i)) *_{v} \\
& =\partial_{j-1}^{1} \partial_{i}^{1}\left(\tau(i)^{*}\right) v=\partial_{j-1}^{1} w_{i}^{1} .
\end{aligned}
$$

It is essential for this computation that $(\tau(i)(j)) * v=(\tau(j)(i))^{*} v$ should already have been determined: It is at this point that the lexicographical order of determination is needed. This completes the discussion of consistency in the lexicographical case; the antilexicographical one is obtained by exchanging the roles of $\nu$ and $\mu$, types 1 and 2, 3 and 4, throughout.

6. Function spaces. In the homotopy theory of spaces, function spaces play an important role. The semisimplicial analogue (originally suggested by A. Heller) is given in Definition 12; Theorem 5 is the analogue of a well-known theorem (due to J.-P. Serre) in the case of spaces. 
Definition 12. If $X, Y$ are semisimplicial complexes, $Y^{X}$ is the semisimplicial complex defined as follows:

(i) $\left(Y^{\boldsymbol{X}}\right)_{q}$ is the set of semisimplicial maps $f: X \times \Delta^{q} \rightarrow Y$, and

(ii) if $f: X \times \Delta^{q} \rightarrow Y, \partial_{i} f: X \times \Delta^{q-1} \rightarrow Y$ and $s_{i} f: X \times \Delta^{q+1} \rightarrow Y$ are defined by

$$
\partial_{i} f=f \circ\left(1 \times \epsilon^{i}\right), \quad s_{i} f=f \circ\left(1 \times \eta^{i}\right) .
$$

Theorem 4. If $Y$ is a Kan-complex, so is $Y^{X}$.

Theorem 5. Let $X, Y$ be a semisimplicial complex, $A$ a subcomplex of $X$ and let $Y$ satisfy the Kan condition. Then the map $p: Y^{X} \rightarrow Y^{A}$ given by $p f$ $=f \mid A \times \Delta^{q}$, where $f: X \times \Delta^{q} \rightarrow Y$, is a fibre map.

To prove Theorem 4 , we consider a nonempty set of less than $q$ maps $f_{i}: X \times \Delta^{q-1} \rightarrow Y, 0 \leqq i \leqq q-1$ such that $\partial_{i} f_{j}=\partial_{j-1} f_{i}$ when $i<j$. We have to construct a map $f: X \times \Delta^{q} \rightarrow Y$ such that $f_{i}=\partial_{i} f$.

The only difference in proving Theorem 5 is that we must assume $f \mid A \times \Delta^{q}$ to be already given.

If $Q$ is any subset of $X$ (not necessarily a subcomplex) we denote by $(Q)$ the subcomplex obtained by applying all suitable semisimplicial operators to elements of $Q$.

We shall define $f \mid\left(X_{n}\right) \times \Delta^{q}$ by an induction on $n$.

To define $f \mid\left(X_{0}\right) \times \Delta^{q}$, it suffices to define $f\left(s_{q-1} \cdots s_{1} s_{0} x, \delta^{q}\right) \in Y$ for every $x \in X_{0}$ : and the possibility of doing this appropriately is easily seen to be precisely the Kan condition on $Y$.

Suppose, then, that $f \mid\left(X_{n-1}\right) \times \Delta^{q}$ has been defined so as to satisfy the required conditions, and let $x \in X_{n}$ be nondegenerate, i.e. $x \in\left(X_{n-1}\right)$. Also, in the case of Theorem 5, let $x \notin A$. Let $\tilde{x}: \Delta^{n} \rightarrow X$ be defined as in Chapter 1, i.e., $\tilde{x} \delta^{n}=x$. We define

$$
\begin{array}{lr}
v_{i}^{1}: \Delta^{n-1} \times \Delta^{q} \rightarrow Y & (i=0, \cdots n), \\
v_{j}^{2}: \Delta^{n} \times \Delta^{q-1} \rightarrow Y & \text { (whenever } f_{j} \text { is given) }
\end{array}
$$

by

$$
\begin{aligned}
& v_{i}^{1}=f \circ\left(\partial_{i}^{1} \tilde{x} \times 1\right)=f \circ\left(\bar{x}^{i} \times 1\right), \\
& v_{j}^{2}=f_{j} \circ(\bar{x} \times 1) .
\end{aligned}
$$

The definition of $v_{i}^{1}$ is legitimate, for the image of $\tilde{x} \epsilon^{i}$ lies in $\left(X_{n-1}\right)$. A simple verification shows that the set $\left\{v_{i}^{1}, v_{j}^{2}\right\}$ is consistent. Hence, by Theorem 3, there is a map

$$
v: \Delta^{n} \times \Delta^{q} \rightarrow Y \text {, i.e. } v \in Y(n, q)
$$

such that

$$
\stackrel{1}{v_{i}}=\partial_{i}^{1} v, \quad v_{j}^{2}=\partial^{2}, v .
$$

We now define $f(x, r)=v\left(\delta^{n}, r\right)$ if $r \in \Delta_{n}^{q}$; more generally, if $\varphi$ is any semi- 
simplicial operator of height $t$ (cf. \$1) we define

$$
f(\varphi x, r)=\left(\varphi^{1} v\right)\left(\delta^{n+t}, r\right) \text { if } r \in \Delta_{n+t}^{q}
$$

where $\varphi^{1}$ denotes the operator obtained by replacing $\partial_{i}$ by $\partial_{i}^{1}, s_{i}$ by $s_{i}^{1}$ in $\varphi$.

This defines $f$ on $(x) \times \Delta^{q}$.

A few straightforward verifications now show that $f$ is a semisimplicial map satisfying all our conditions; which moreover agrees with the inductively given map on $\left(X_{n-1}\right) \times \Delta^{q}$.

Proceeding similarly with every nondegenerate element of $X_{n}-A$, our induction is complete.

Appendix I: Proof of Lemma 3. Let $\sigma=\left(\nu_{1}, \cdots, \nu_{q} ; \mu_{1}, \cdots, \mu_{p}\right)$, $\tau=\left(\bar{\nu}_{1}, \cdots, \bar{\nu}_{q} ; \bar{\mu}_{1}, \cdots, \bar{\mu}_{p}\right)$.

(i) Let $(i, r, s)$ be of $\sigma$-type 1 . Then

$$
\partial_{i} \delta_{\sigma}^{p+q}=\left(s_{\nu_{q}-1} \cdots s_{\nu_{r+1}-1} s_{\nu_{r}} \cdots s_{\nu_{1}} \partial_{i-r} \delta^{p}, s_{\mu_{p}-1} \cdots s_{\mu_{g}+1-1} s_{\mu_{s-1}} \cdots s_{\mu_{1}} \delta^{q}\right)
$$

and it is easily seen that $\partial_{j} \delta_{\tau}^{p+\ell}$ has a different type of expression unless $(j, \bar{r}, \bar{s})$ is of $\tau$-type 1 . Hence, by Lemma $1,(j, \bar{r}, \bar{s})$ is of $\tau$-type 1 .

If $0 \leqq i \leqq p+q$ define $X_{\sigma}(i)=i-t$ where $t$ is the largest integer such that $\nu_{t}<i\left({ }^{2}\right)$. Clearly $i \leqq j$ implies $X_{\sigma}(i) \leqq X_{\sigma}(j)$. If $(i, r, s)$ is of $\tau$-type $1, X_{\sigma}(i)$ $=i-r$; hence by Lemma 1 and the expressions for $\partial_{i} \delta_{\sigma}^{p+q}, \partial_{j} \delta_{\tau}^{p+q}$ we must have $X_{\sigma}(i)=i-r=j-\bar{r}=X_{\tau}(j)$. Now suppose $\bar{r}<r$. Then $j<i$ and, again by Lemma 1,

$$
\begin{array}{lcc}
\nu_{1}=\bar{\nu}_{1} & \nu_{\bar{r}+1}=\bar{\nu}_{\bar{r}+1}-1 & \nu_{r+1}=\bar{\nu}_{r+1} \\
\hdashline \nu_{\bar{r}}=\bar{\nu}_{r} & \nu_{r}=\bar{\nu}_{r}-1 & \nu_{q}=\bar{\nu}_{q}
\end{array}
$$

Also, $X_{\sigma}(i)=X_{\sigma}(i-1)+1 \geqq X_{\sigma}(j+1)+1>X_{\sigma}(j)$, for $j<\bar{\nu}_{r+1}=\nu_{r+1}+1 \leqq \nu_{r}+1$ $<i$ whence $j+1 \leqq i-1$. Since $\nu_{r}=\bar{\nu}_{\bar{r}}, \nu_{\bar{r}+1}=\bar{\nu}_{r+1}-1 \geqq j$ we have $X_{\sigma}(j)=j-\bar{r}$ $=X_{\tau}(j)$. Hence $X_{\tau}(j)<X_{\sigma}(i)$, and we have a contradiction. Similarly $\bar{r}>r$ is impossible, whence $\bar{r}=r$ and $i=j$. From which with Lemma 1 , the result is immediate. The case when $i$ is of $\tau$-type 2 is proved similarly.

(ii) Let $(i, r, s)$ be of $\tau$-type 3 or 4 . Then again it is immediate that $(j, \bar{r}, \bar{s})$ must also be of $\sigma$-type 3 or 4 .

$$
\partial_{i} \delta_{\sigma}^{p+q}=\left(s_{\alpha_{q-1}} \cdots s_{\alpha_{1}} \delta^{p}, s_{\beta_{p-1}} \cdots s_{\beta_{1}} \delta^{q}\right)
$$

where

$$
\begin{array}{ll}
\alpha_{1}=\nu_{1} & \beta_{1}=\mu_{1} \\
\alpha_{r-1}=\nu_{r-1} & \beta_{s-1}=\mu_{s-1} \\
\alpha_{r}=\nu_{r+1}-1 & \beta_{s}=\mu_{s+1}-1 \\
\alpha_{q-1}=\nu_{q}-1 & \beta_{p-1}=\mu_{p}-1
\end{array}
$$

(2) $t=0$ if $i<\nu_{1}$. 
Since $\sigma$ is a $(p, q)$ shuffle we see that $\left(\alpha_{1}, \cdots, \alpha_{q-1} ; \beta_{1} \cdots \beta_{p-1}\right)$ is a permutation of $[p+q-2]-\{i-1\}$. Hence the index $i-1$ is uniquely implied by a knowledge of the $\alpha$ 's and $\beta$ 's i.e. (by Lemma 1 ) by $\partial_{i} \delta_{\sigma}^{p+q}$. Hence $i=j$.

Moreover, $\left(\alpha_{1}, \cdots, \alpha_{q-1} ; \beta_{1}, \cdots, \beta_{p-1}\right)$ is obtained from $\left(\nu_{1}, \cdots, \nu_{q}\right.$; $\left.\mu_{1}, \cdots, \mu_{p}\right)$ by removing $i-1$ and $i$ and decreasing the integers $>i$ by 1 . Hence $\left(\bar{\nu}_{1}, \cdots, \bar{\nu}_{q} ; \bar{\mu}_{1}, \cdots, \bar{\mu}_{p}\right)$ can differ from $\left(\nu_{1}, \cdots, \nu_{q} ; \mu_{1}, \cdots, \mu_{p}\right)$ only in respect of $i$ and $i-1$. Hence $\tau=\sigma$ or $\tau=\sigma(i)$.

Appendix II: Prisms. We write $I=\Delta^{1}$ and define $I^{r}$ by $I^{1}=I, I^{r+1}=I^{r} \times I$. If $A$ is a semisimplicial complex we shall write $A_{r, n}$ for $A(1, \cdots, 1, n)$, i.e. the set of semisimplicial maps $I^{r} \times \Delta^{n} \rightarrow A$. By $h_{i}: A(1, \cdots, 1,0,1, \cdots, 1, n)$ $\rightarrow A_{r-1, n}$ (where " 0 " stands in the $i$ th place) we denote the obvious identification. Then we define

$$
\lambda_{i}^{\epsilon}: A_{r, n} \rightarrow A_{r-1, n} \quad(\epsilon=0,1 ; 1 \leqq i \leqq r)
$$

by

$$
\lambda_{i}^{e}=h_{i} \partial_{1-\epsilon}^{i}
$$

and

$$
\sigma_{i}: A_{r-1, n} \rightarrow A_{r, n} \quad(1 \leqq i \leqq r)
$$

by

$$
\sigma_{i}=s_{0}^{i}\left(h_{i}\right)^{-1} \text {. }
$$

Finally, we define $\partial_{i}: A_{r, n} \rightarrow A_{r, n-1}, s_{i}: A_{r, n} \rightarrow A_{r, n+1}$ by $\partial_{i}=\partial_{i}^{r+1}, s_{i}=s_{i}^{r+1}$.

It is now easily verified that these operators satisfy all the conditions of the "prismatic theory," cf. [3], Chapter III. In particular, putting $n=0$ and ignoring $\partial$ and $s$ we obtain a semi-cubical theory: If $A$ satisfies Kan's condition so, by Theorem 3, does the resulting semi-cubical complex.

If we write $\tau_{i}$ for the $(1, n)$-shuffle $(0, \cdots, i-1, i+1, \cdots, n ; i)$ $(0 \leqq i \leqq n)$, we can define $S_{i}=\tau_{i}^{*}: A_{r, n} \rightarrow A_{r-1, n+1}$, where we modify our earlier process by combining the first and last index (using $\tau_{i}$ ), rather than the first and second.

It is now an easy matter to verify all the relations given for the separtition operators in Chapter III of [3]. (The relations involving boundaries are, of course, special cases of $3^{*}, 5^{*}, 6$ above).

We thus obtain the complete formalism of the semiprismatic theory of [3], and in particular the theory of homotopy there given; cf. also Exp. 14 of [5] where the same notion of semisimplicial homotopy is used.

Using Theorem 3, it is easy to prove a homotopy extension theorem for Kan complexes, cf. $[1 ; 2]$.

\section{RefERENCES}

1. D. M. Kan, Abstract homotopy I, Proc. Nat. Acad. Sci. U.S.A. vol. 41 (1955) p. 1092. 
2. - Abstract homotopy II, Proc. Nat. Acad. Sci. U.S.A. vol. 42, p. 255.

3. V. K. A. M. Gugenheim and J. C. Moore, Acyclic models and fibre spaces, Trans. Amer. Math. Soc. (to appear in 1957).

4. S. Eilenberg and S. MacLane, On the groups $H(\pi, n)$, I, Ann. of Math. vol. 58 (1953) p. 55.

5. H. Cartan, Seminaire, 1954-1955 (polycopied).

Birkbeck College,

LONDON, ENGLAND 